\title{
Pitch Guidance Optimization for the Orion Ascent Abort Flight Tests
}

\author{
Ryan Stillwater \\ NASA Dryden Flight Research Center \\ 2010 AIAA Atmospheric Flight Mechanics Conference \\ 2-5 August 2010 \\ Toronto, Ontario, Canada
}




\section{Introduction}

- Orion vehicle overview

- Orion abort flight tests overview

- What is being optimized

- Method used for optimization

- Results from the optimization

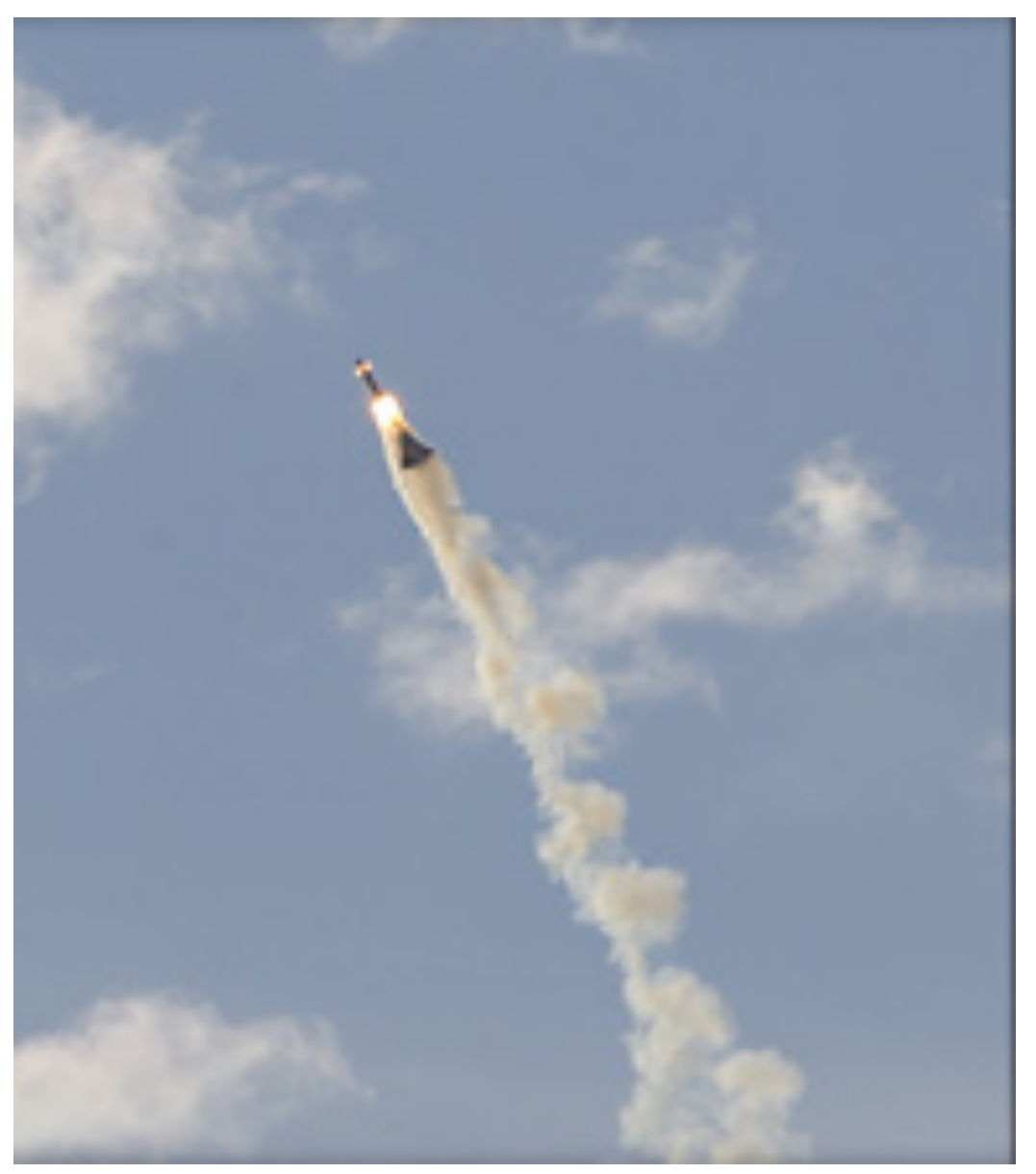




\section{Constellation Overview}

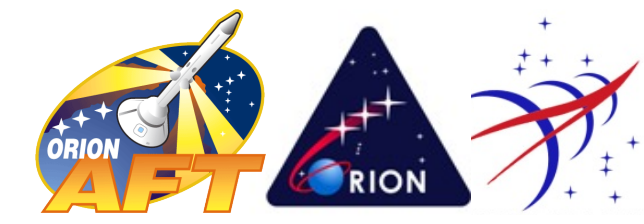

- Constellation program was initiated to create the next manned space vehicle

- Ares I launch vehicle

- Launches Orion crew vehicle into orbit

- Orion crew vehicle

- Carries astronauts to ISS or Moon

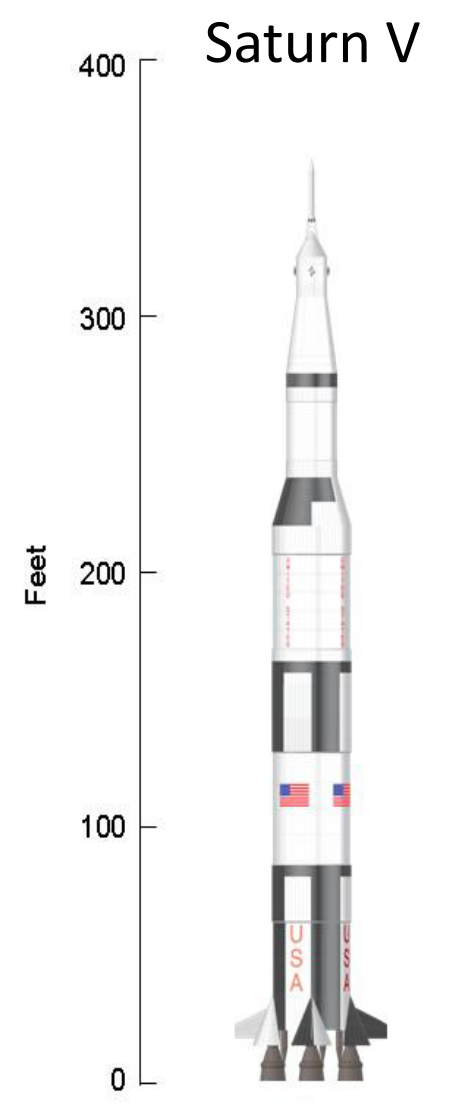

Ares I

Shuttle

Orbiter
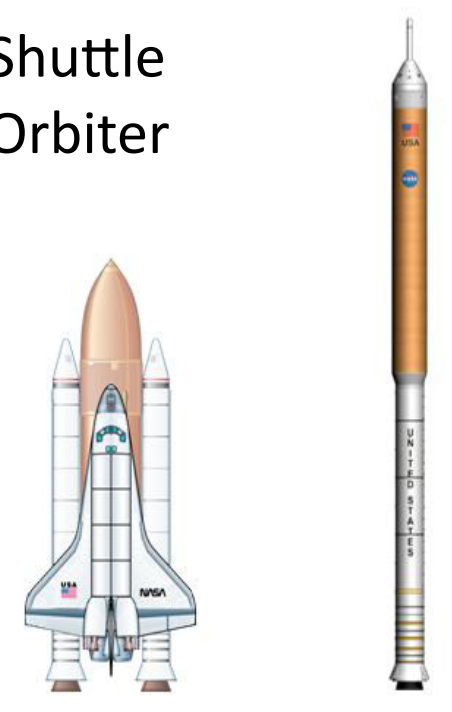


\section{Orion Vehicle Overview}

- Launch Abort System (LAS)

- Will remove the CM from the Ares I in the event of a launch failure

- Crew Module (CM)

- Carries 6 crew to the ISS or 4 crew to the Moon

- 5 meter diameter (Apollo was 3.9 meter)

- Launch Abort Vehicle (LAV)

- Combined CM and LAS

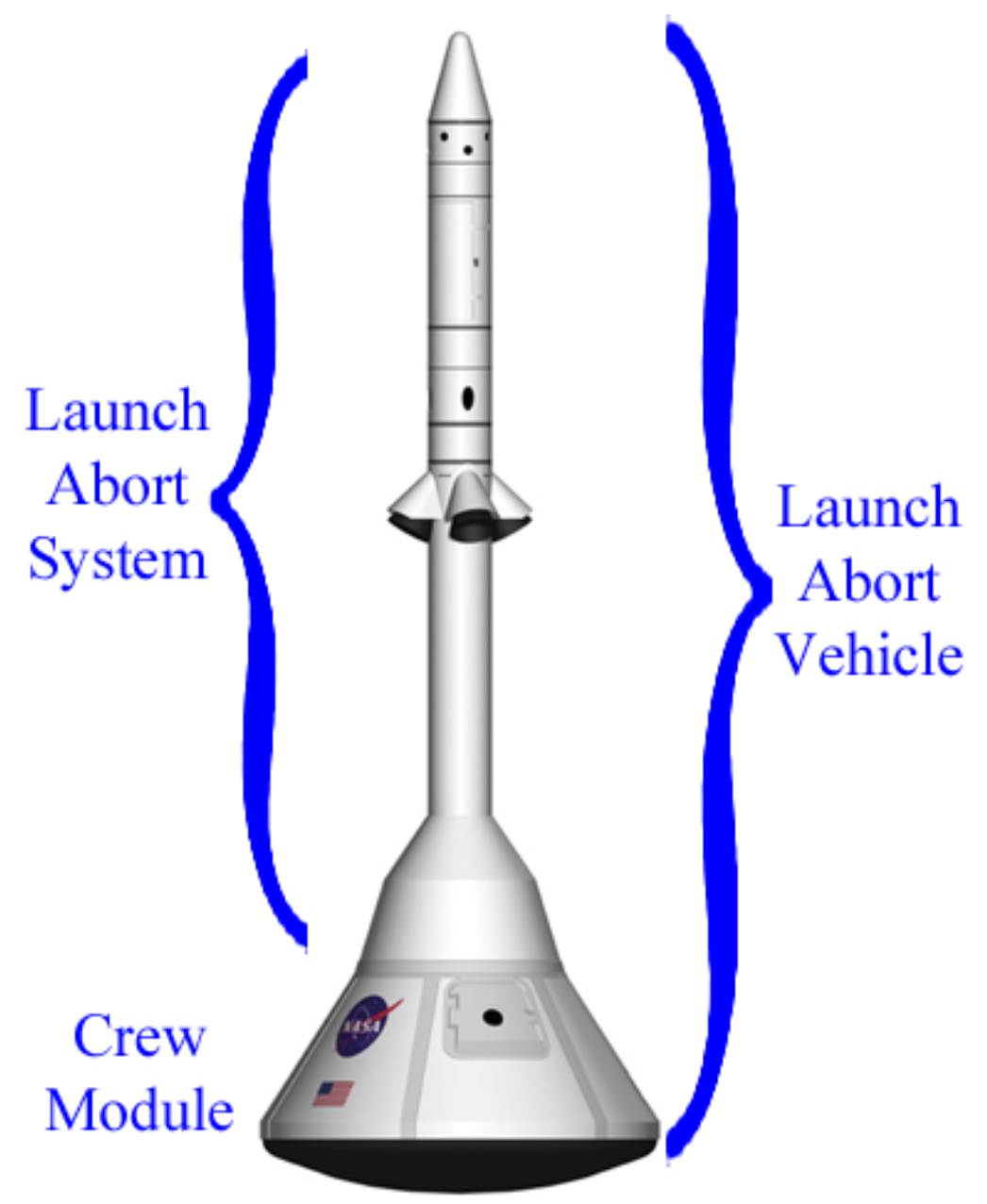




\section{LAS Overview}

- The Launch Abort System (LAS) will rescue the crew in the event of a launch vehicle failure

- Consists of three solid rocket motors

- Abort Motor (AM)

- Ignites on abort

- Provides thrust to separate the Launch Abort Vehicle and the Ares I

- Attitude Control Motor (ACM)

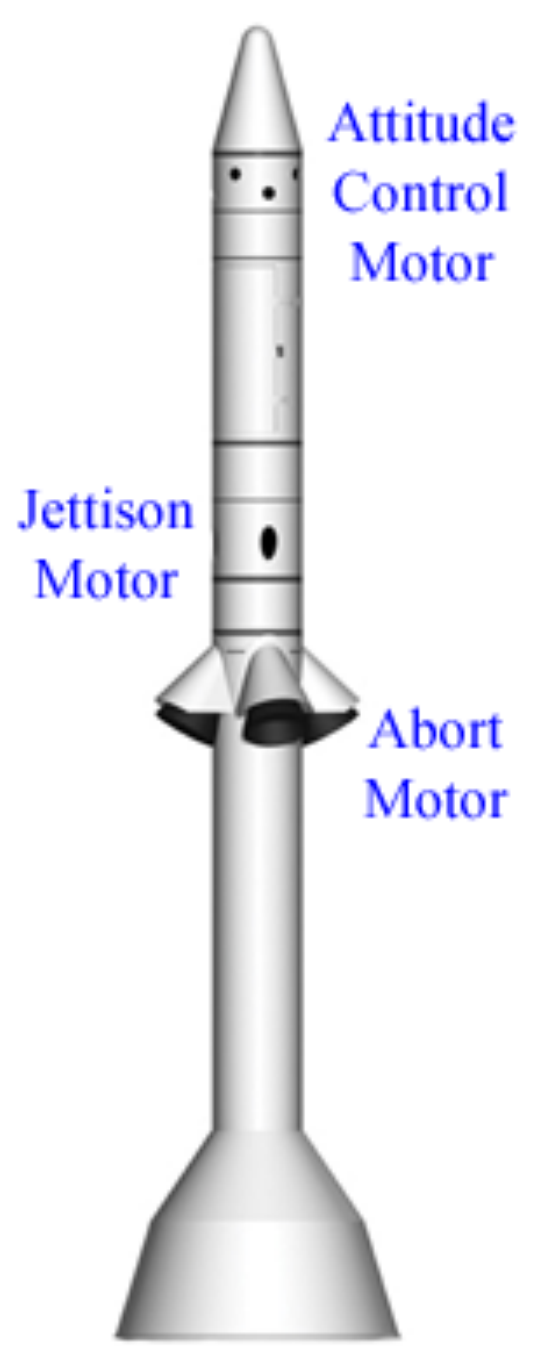

- Separates the LAS from the CM 


\section{LAS Controller}

- PID controller with channels for the pitch and yaw axes

- Uses time-based gain schedules

- Pitch channel

- Uses angle of attack $(\alpha)$, integral $\alpha$, pitch angle $(\theta)$, pitch rate $(q)$, and flight path angle $(\gamma)$

- Used for optimization

- Yaw channel

- Uses sideslip angle $(\beta)$, integral $\beta$, yaw angle $(\psi)$, yaw rate $(r)$, roll angle $(\phi)$, roll rate $(p)$, and heading

- Also commands $\beta$ to damp out initial roll rate using an aerodynamic roll moment

- Not used for optimization

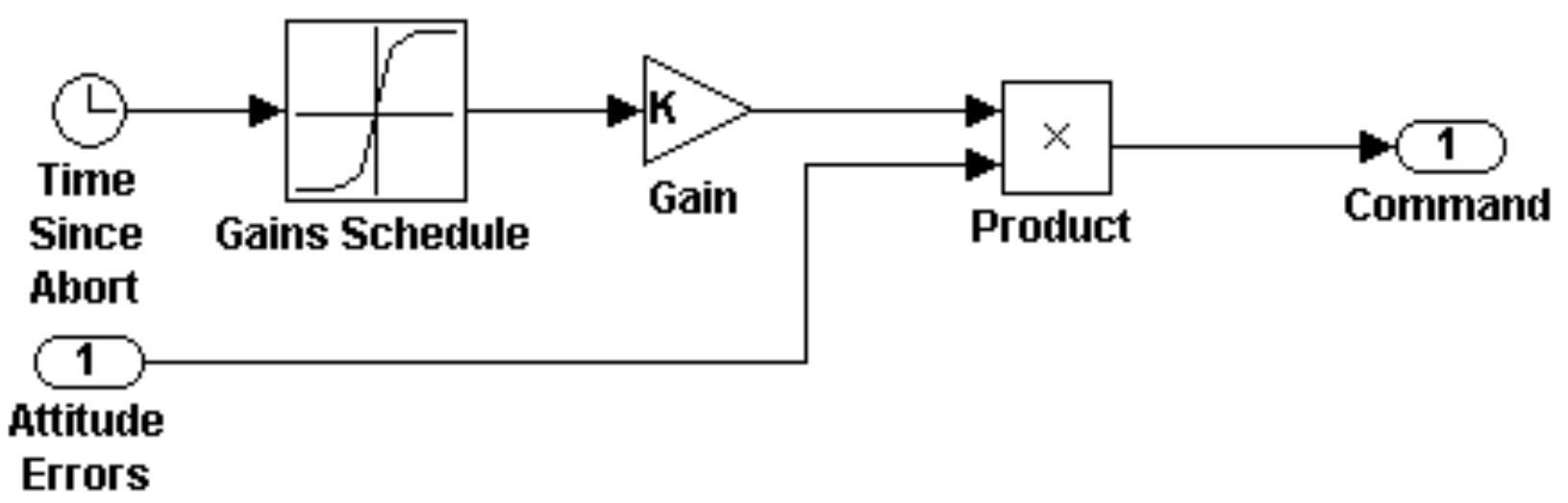




\section{Orion Abort Flight Tests}

- Six flight tests were originally scheduled to verify the functionality of the LAS

- Two aborts from the launch pad

- Four aborts along the ascending trajectory

- Uses an Abort Test Booster (ATB) to reach test condition

- Minimum separation force

- Transonic region

- Nominal maximum dynamic pressure

- Maximum dynamic pressure region

- Failure scenario: Ares I nozzle actuators stick hard-over

- High dynamic pressure region

- High altitude

- Stage 1 burnout/Stage 2 ignition point 


\section{Test Regions}

- Three ascent abort test regions used for optimization

- Transonic

- Maximum dynamic pressure

- Ares I nozzle failure at high dynamic pressure

- Stage $1 /$ Stage 2 separation was not included

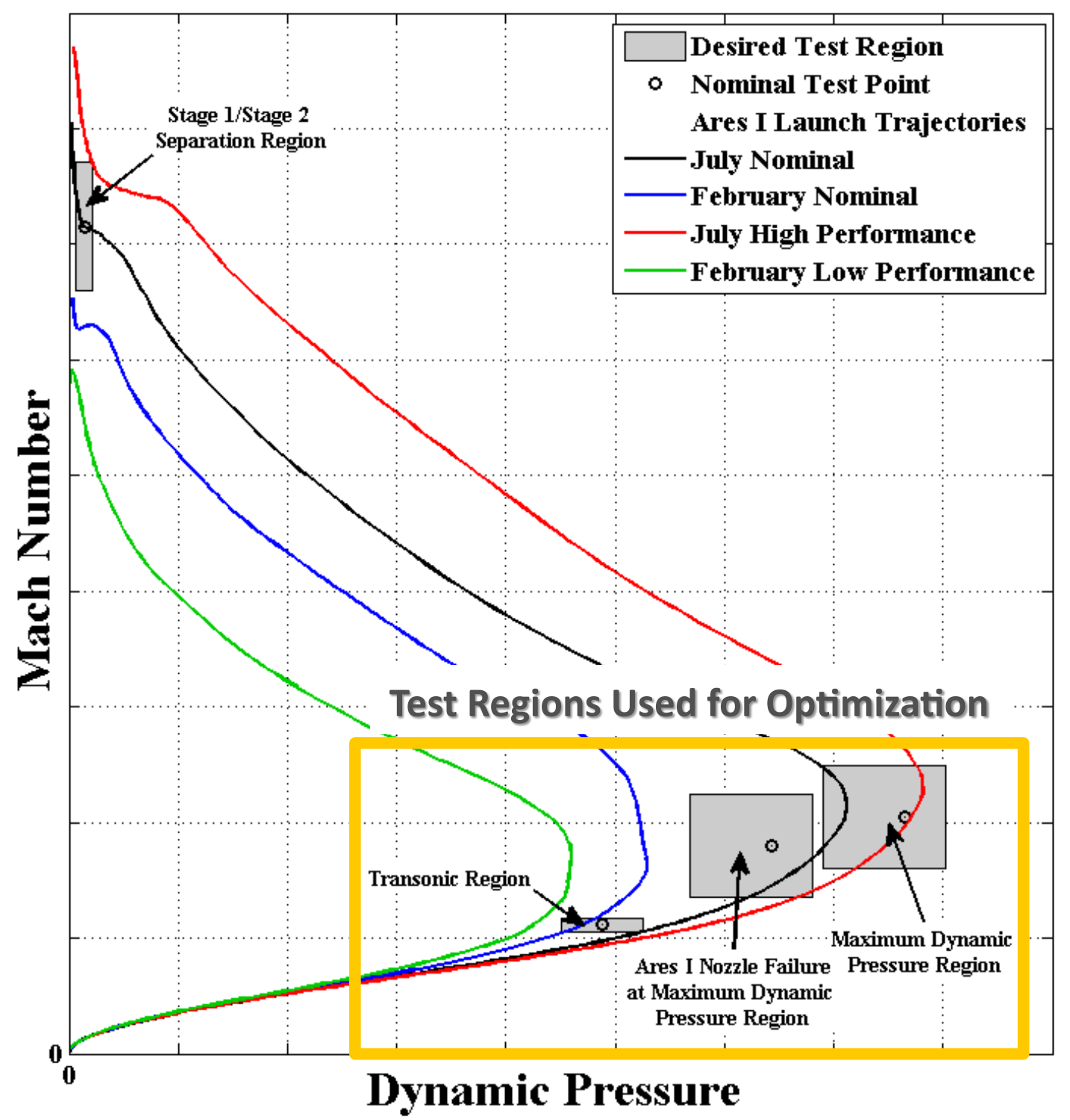




\section{Ascent Abort ConOps}

\begin{tabular}{|c|l|}
\hline \multicolumn{2}{l}{} \\
\hline Event & \multicolumn{1}{|c|}{ Event } \\
\hline $\mathbf{1}$ & ATB Liftoff \\
\hline $\mathbf{2}$ & LAV Separation \\
\hline $\mathbf{3}$ & Begin Reorientation \\
\hline $\mathbf{4}$ & End Reorientation \\
\hline $\mathbf{5}$ & Jettison Tower \\
\hline $\mathbf{6}$ & Deploy Drogues \\
\hline $\mathbf{7}$ & Deploy Mains \\
\hline $\mathbf{8}$ & CM Touchdown \\
\hline
\end{tabular}

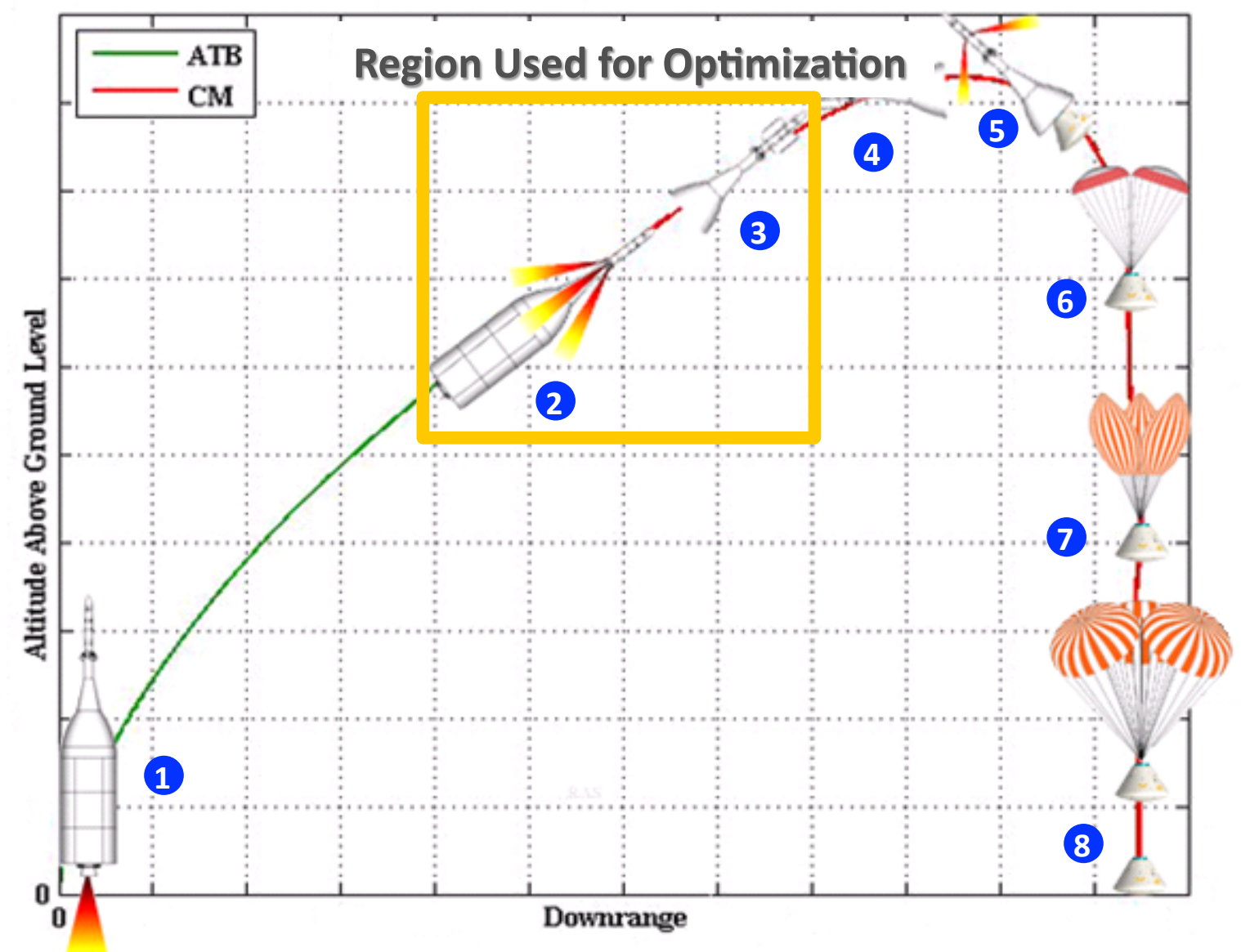




\section{Simulation Overview}

- ATB/LAV Simulation

- Created by Orbital Sciences Corporation (Chandler, AZ)

- Simulations developed for the three test regions used

- Simulates ATB/LAV vehicle dynamics up thru ATB/LAV separation

- Provides initial conditions for LAV simulation

- LAV Simulation

- Created by NASA

- Initialized at ATB/LAV separation point

- Generates the state data used in optimization program

- Used to simulate vehicle dynamics until LAS/CM separation 


\section{Problem Statement}

- Problem Statement

- Tune the Orion pitch gains schedule to reduce the error between the simulated $\alpha$ and the desired $\alpha$

- Cost Function

- Running the LAV simulation with a different gains schedule results in different $\alpha$ errors $\left(\alpha_{\mathrm{e}}\right)$

$$
\alpha_{e}=f\left(K_{\alpha}, K_{\text {integral } \alpha}, K_{\theta}, K_{q}, K_{\gamma}\right)
$$

- The optimization program seeks where the gradient of $\alpha_{e}$ is zero (minima) 


\section{Optimization Algorithm}

- Uses the method of steepest descent

- Outer loop

- Approximate Jacobian

$$
\bar{J}\left(\overline{K_{n, 1}}\right)=\left[\begin{array}{c}
\frac{\delta f}{\delta K_{\alpha_{n, 1}}} \\
\frac{\delta f}{\delta K_{i \alpha_{n, 1}}} \\
\frac{\delta f}{\delta K_{q_{n, 1}}} \\
\frac{\delta f}{\delta K_{\theta_{n, 1}}} \\
\frac{\delta f}{\delta K_{\gamma_{n, 1}}}
\end{array}\right]
$$$$
\overline{K_{n, 1}}+\Delta K_{\alpha}=\left[\begin{array}{c}
K_{\alpha_{n, 1}} \\
K_{i \alpha_{n, 1}} \\
K_{q_{n, 1}} \\
K_{\theta_{n, 1}} \\
K_{\gamma_{n, 1}}
\end{array}\right]+\left[\begin{array}{c}
\Delta K_{\alpha} \\
0 \\
0 \\
0 \\
0
\end{array}\right], \quad \overline{K_{n, 1}}-\Delta K_{\alpha}=\left[\begin{array}{c}
K_{\alpha_{n, 1}} \\
K_{i \alpha_{n, 1}} \\
K_{q_{n, 1}} \\
K_{\theta_{n, 1}} \\
K_{\gamma_{n, 1}}
\end{array}\right]-\left[\begin{array}{c}
\Delta K_{\alpha} \\
0 \\
0 \\
0 \\
0
\end{array}\right]
$$$$
\frac{\delta f}{\delta K_{\alpha_{n, 1}}} \approx \frac{f\left(\overline{K_{n, 1}}+\Delta K_{\alpha}\right)-f\left(\overline{K_{n, 1}}-\Delta K_{\alpha}\right)}{2 \Delta K_{\alpha}}
$$ 


\section{Optimization Algorithm}

- Inner loop

- Each time point in gains schedule

Step 1: $\quad \mathrm{c}_{0}=0 \quad \alpha_{e_{0}}=f\left(\overline{K_{n, j}}\right) \quad g_{0}=\sum_{m=1}^{m=31}\left[\alpha_{e_{0}}\right]^{2}$

Step 2: $\quad \nabla g_{0}=2 * \bar{J}\left(\overline{K_{n, 1}}\right) * \alpha_{e_{0}} \quad \bar{z}=\frac{\nabla g_{0}}{\left\|\nabla g_{0}\right\|}$

Step 3: $\quad c_{2}=1 \quad \alpha_{e_{2}}=f\left(\overline{K_{n, j}}-c_{2} * \bar{z}\right) \quad g_{2}=\sum_{m=1}^{m=31}\left[\alpha_{e_{2}}\right]^{2}$

Step 4: if $g 2>g 0$, then rerun Step 3 with $c_{2}=c_{2} / 2$ until $g 2<g 0$ 


\section{Optimization Algorithm}

Step 5: $\quad \mathrm{c}_{1}=\mathrm{c}_{2} / 2 \quad \alpha_{e_{1}}=f\left(\overline{K_{n, j}}-c_{1} * \bar{z}\right) \quad g_{1}=\sum_{m=1}^{m=31}\left[\alpha_{e_{1}}\right]^{2}$

Step 6: $h_{0}=\frac{g_{1}-g_{0}}{c_{1}-c_{0}} \quad h_{1}=\frac{g_{2}-g_{1}}{c_{2}-c_{1}} \quad h_{2}=\frac{h_{1}-h_{0}}{c_{2}-c_{0}}$

Step 7: $\quad P\left(c_{3}\right)=g_{0}+h_{0} * c_{3}+h_{2} * c_{3} *\left(c_{3}-c_{1}\right)$
$P^{\prime}\left(c_{3}\right)=h_{0}+2 * h_{2} * c_{3}-c_{1} * h_{2}$

Step 8: $\quad c_{3}=\frac{c_{1} * h_{2}-h_{0}}{2 * h_{2}} \quad \overline{K_{n, j}+1}=\overline{K_{n, j}}-c_{3} * \bar{z}$

Next inner loop iteration (next gains schedule point)

Convergence check

Next outer loop iteration 


\section{Results}

- Desired $\alpha$ profile for all scenarios was zero $\alpha$ from LAV separation until reorientation

- Assessing the improvement (if any) was performed by:

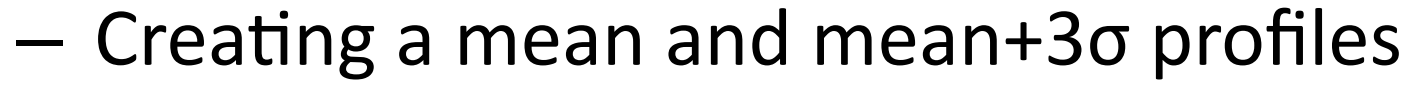

- Gaussian mean and $\sigma$ are calculated at each discrete point

- Differencing the optimized and baseline profiles

- Summing the $\alpha_{e}$ along the profiles for overall change
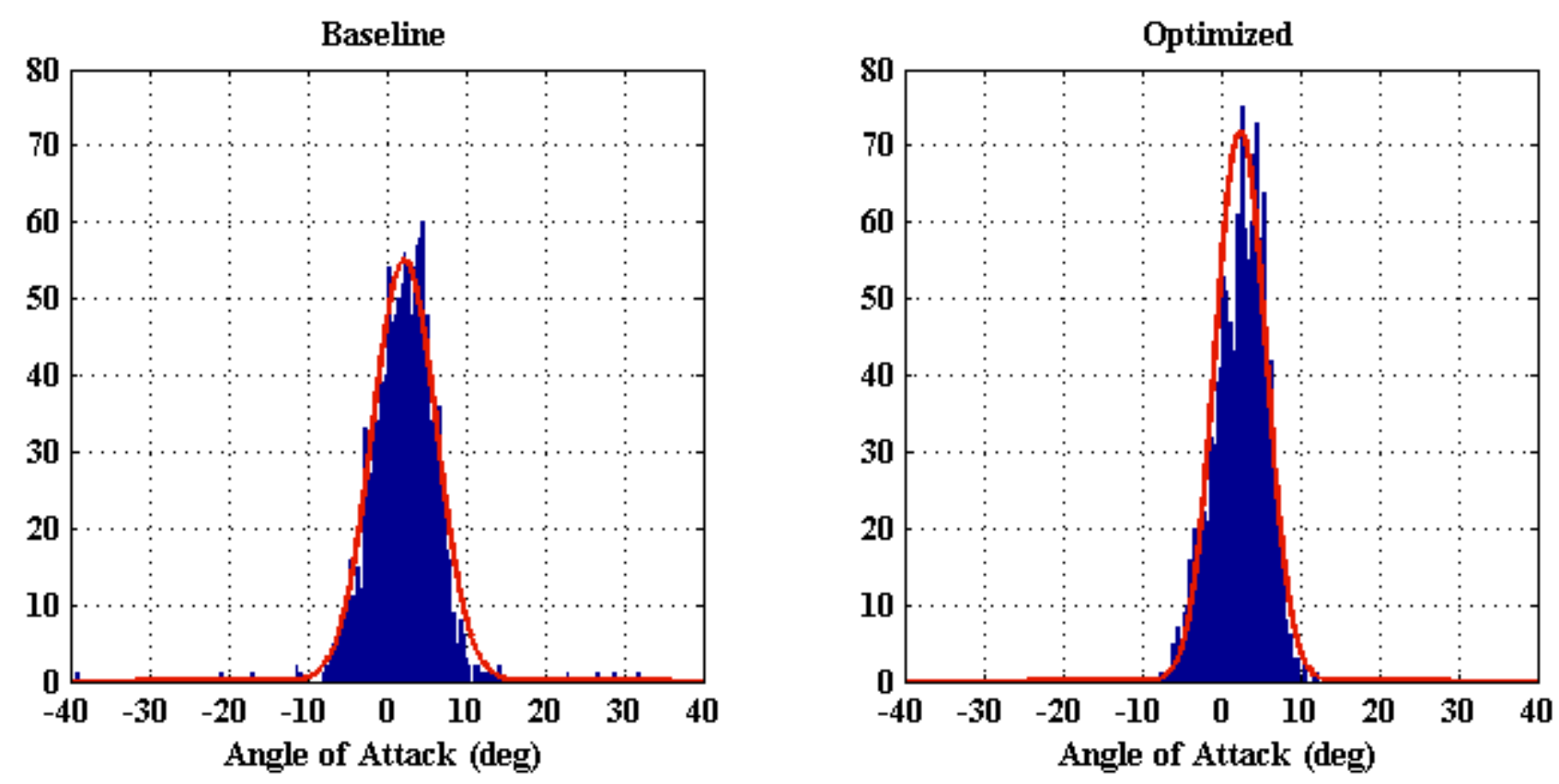


\section{Results}

- Transonic scenario

- Reduced tumbling cases by $24 \%$

- Reduced nominal profile $\alpha_{\mathrm{e}}$ by $49 \%$

- Reduced mean profile $\alpha_{e}$ by $5 \%$

- Reduced mean+3o profile $\alpha_{e}$ by $45 \%$

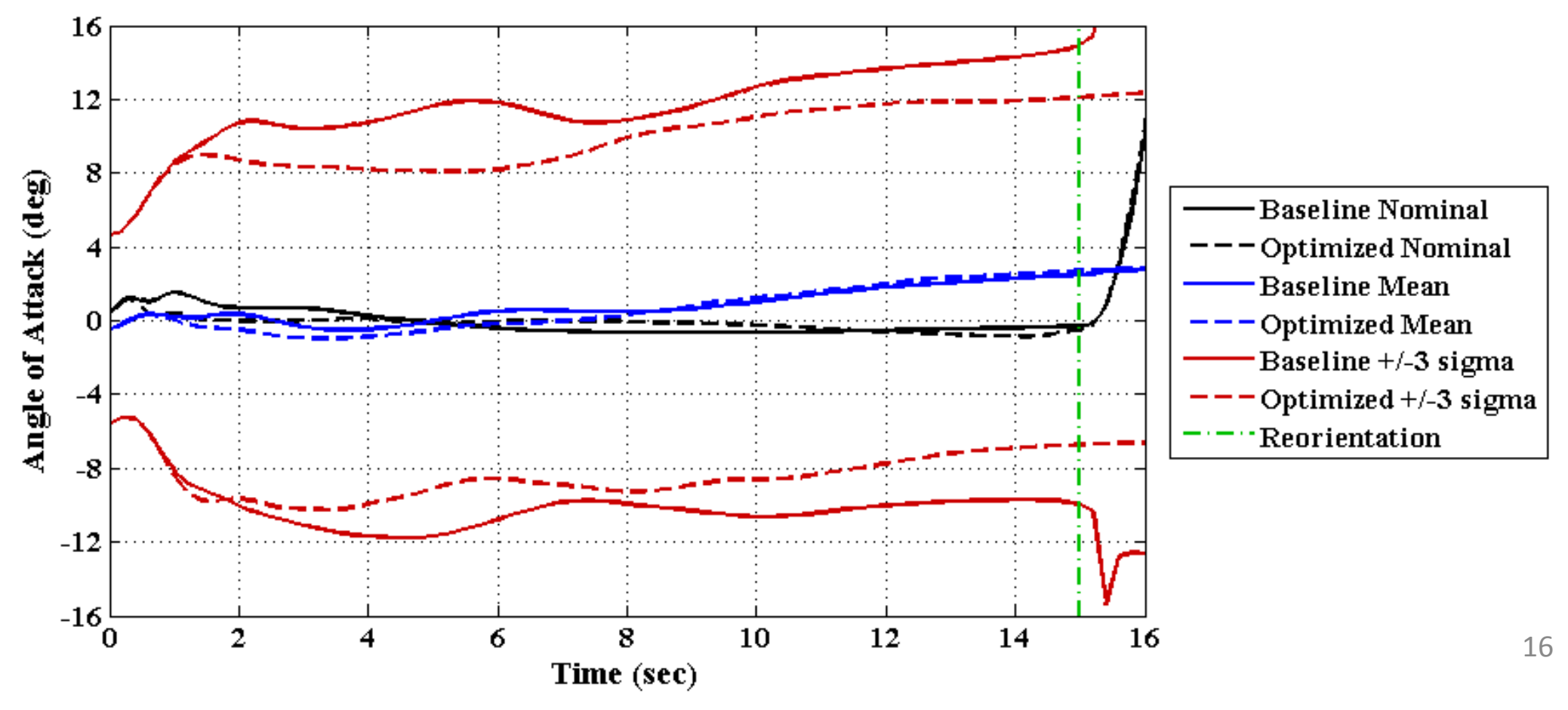




\section{Results}

- Maximum dynamic pressure scenario

- Reduced tumbling cases by $3 \%$

- Reduced nominal profile $\alpha_{\mathrm{e}}$ by $57 \%$

- Reduced mean profile $\alpha_{\mathrm{e}}$ by $28 \%$

- Reduced mean+3o profile $\alpha_{e}$ by $61 \%$

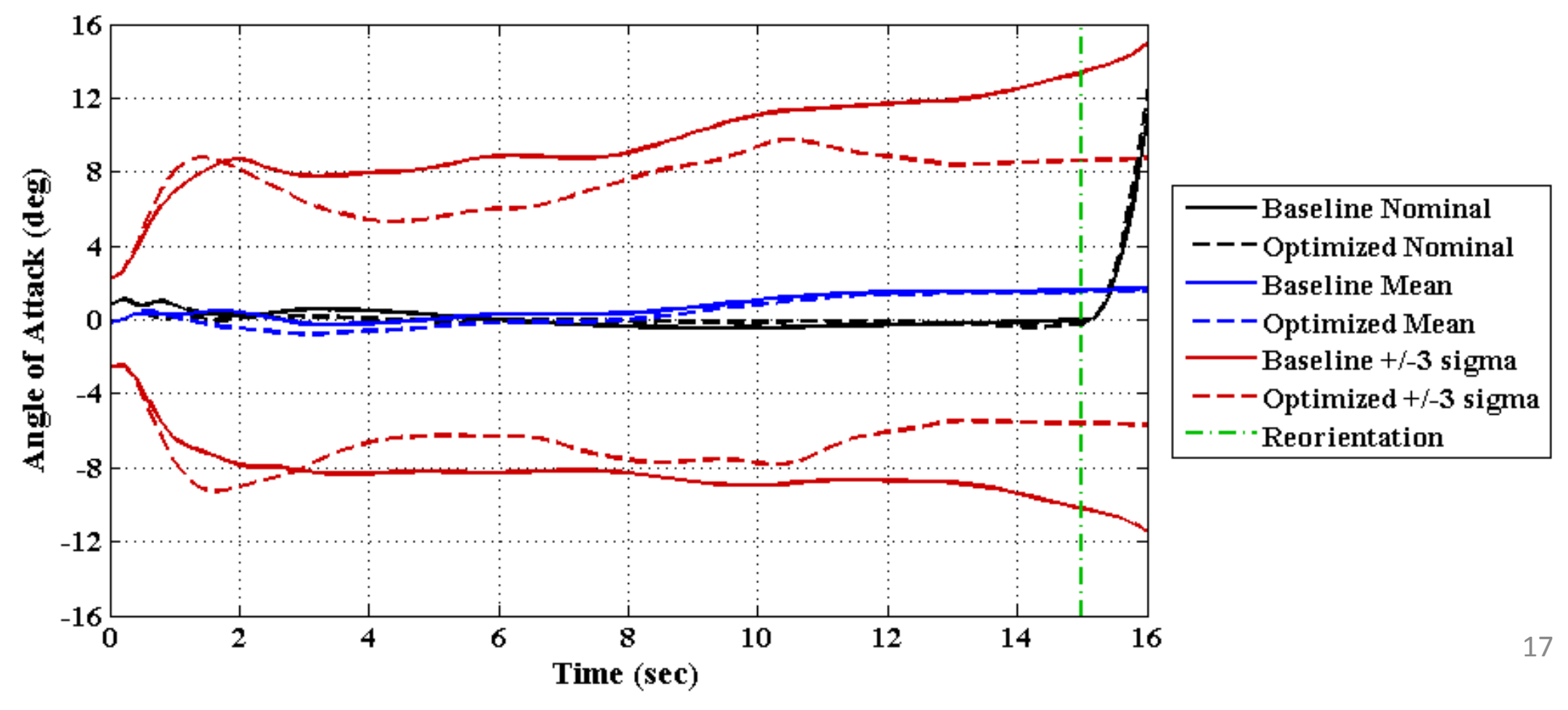




\section{Results}

- Ares I nozzle failure scenario

- Reduced tumbling cases by $47 \%$

- Reduced nominal profile $\alpha_{\mathrm{e}}$ by $35 \%$

- Reduced mean profile $\alpha_{e}$ by $37 \%$

- Reduced mean+3o profile $\alpha_{e}$ by $60 \%$

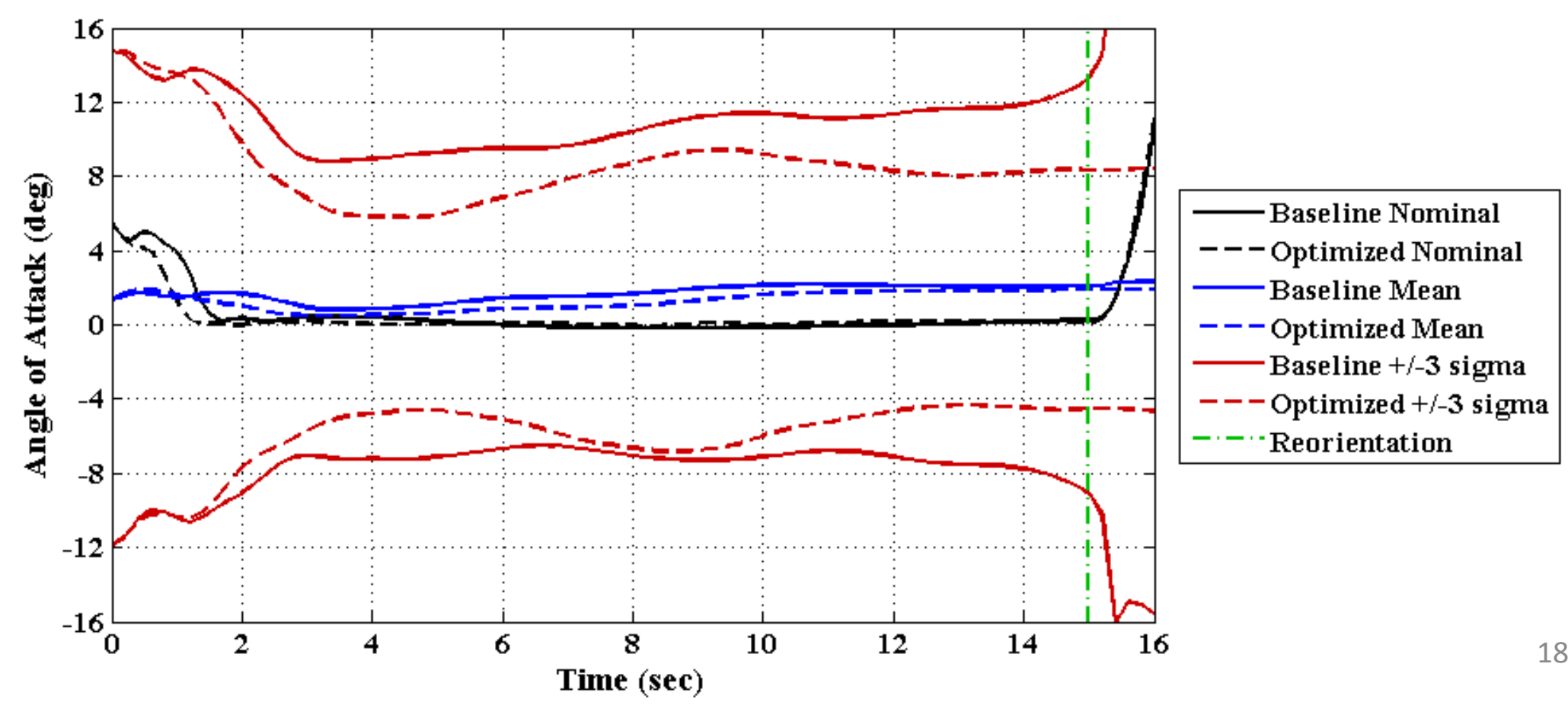




\section{Summary}

- All three scenarios showed overall reduction in the $\alpha$ errors

- The method of steepest descent is effective in tuning the gains schedule

- All Orion ascent abort flight tests should benefit from tuning the gains schedule

- Questions? 


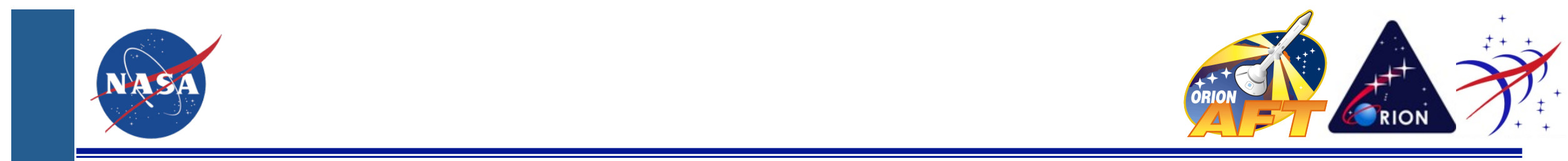

Backup 


\section{Results}

- Transonic scenario

- Nominal profile improved from 0-12 sec

- Mean profile improved mainly from 5-8 sec

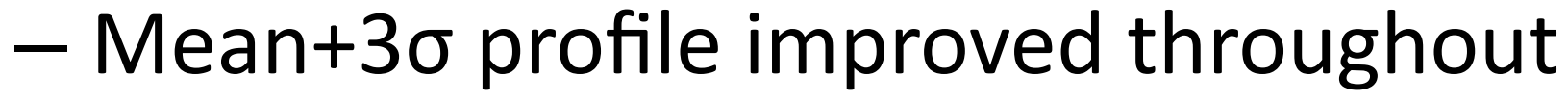

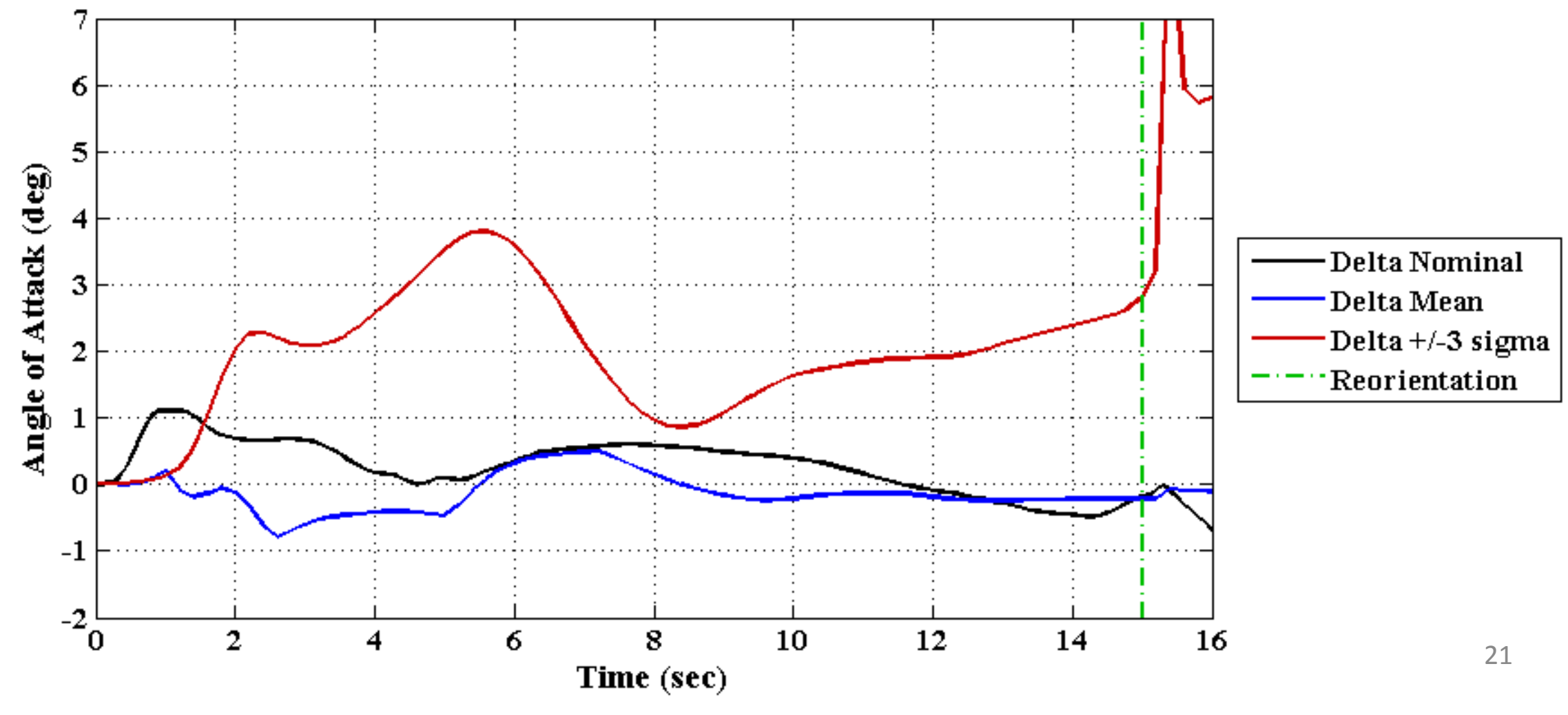




\section{Results}

- Maximum dynamic pressure scenario

- Nominal profile improved from 0-13 sec

- Mean profile improved from 0-2 sec and 5-15 sec

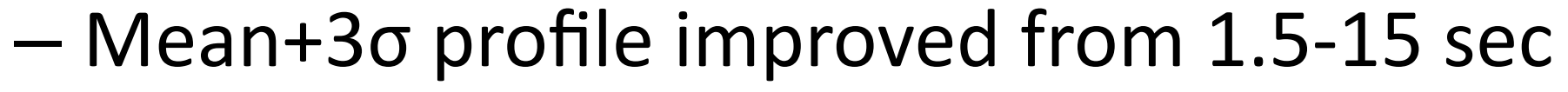

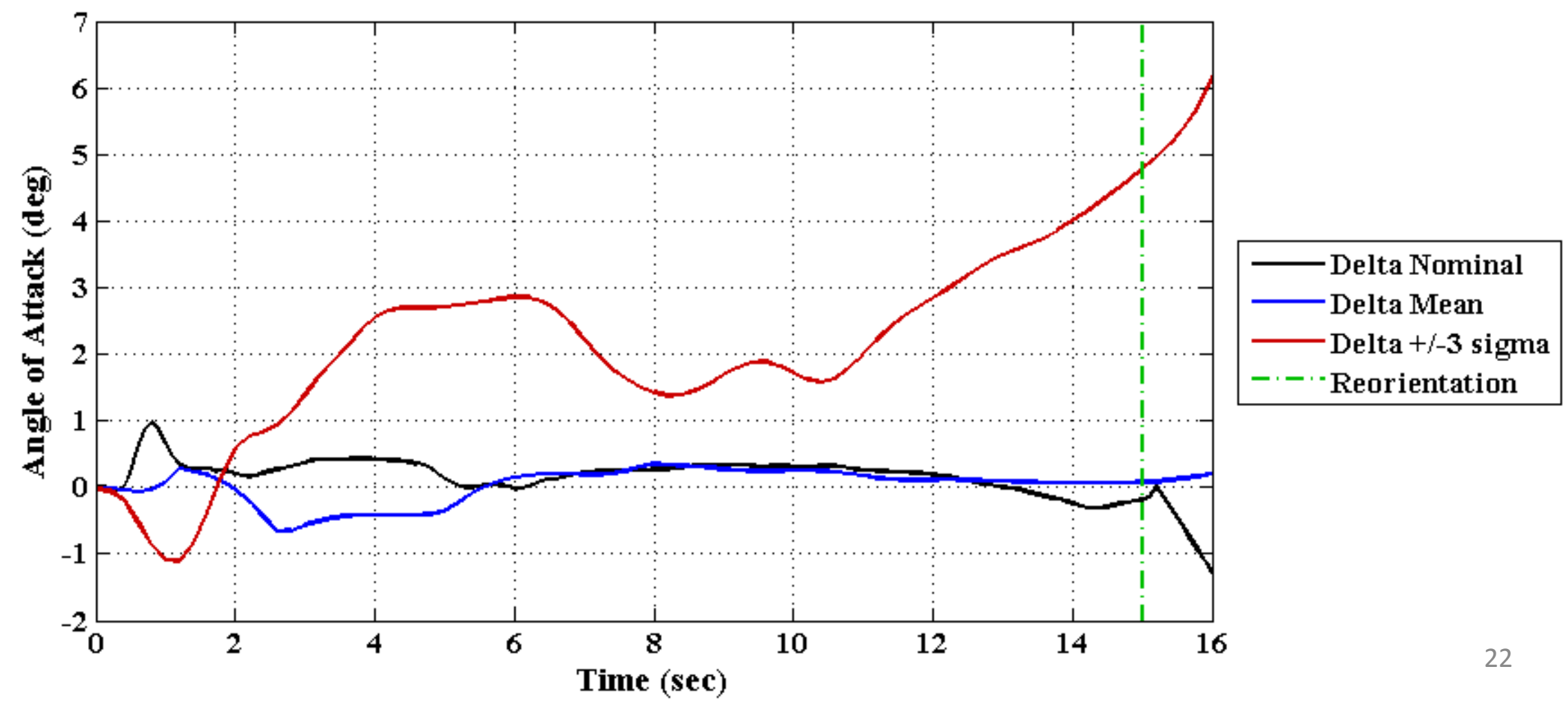




\section{Results}

- Ares I nozzle failure scenario

- Nominal profile improved from 0-11 sec

- Mean profile improved from 1-15 sec

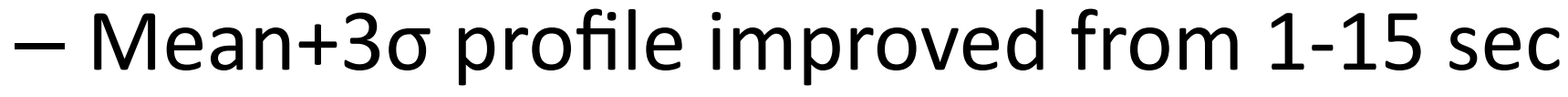

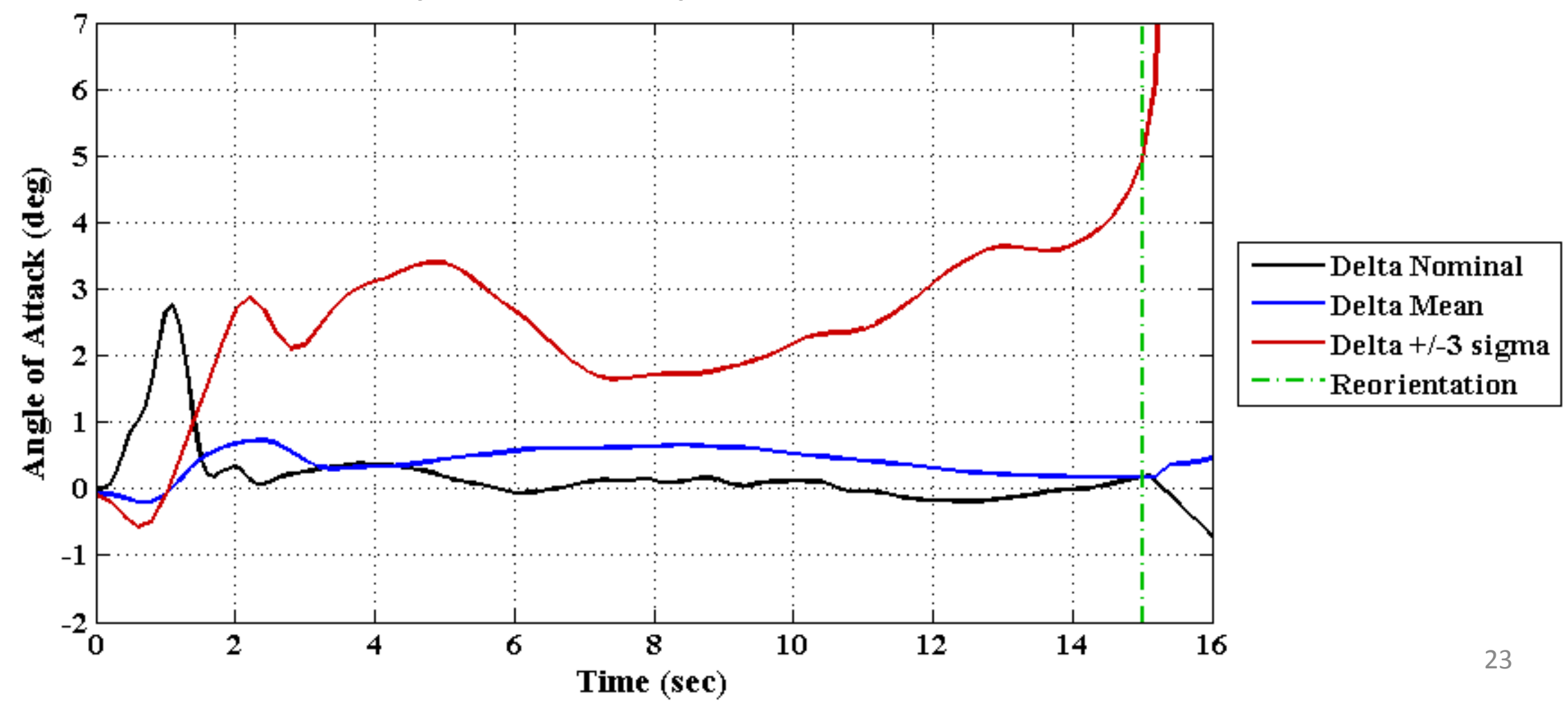

